\title{
A new approach for thin-walled member analysis in the framework of GBT
}

\author{
Gianluca Ranzi ${ }^{\text {a,* }}$, Angelo Luongo ${ }^{\mathrm{b}}$ \\ a School of Civil Engineering, The University of Sydney, NSW 2006, Australia \\ ${ }^{\mathrm{b}}$ Dipartimento d'Ingegneria delle Strutture, delle Acque e del Terreno, Università dell'Aquila, Italy
}

\begin{abstract}
A B S T R A C T
A new approach is illustrated for the cross sectional analysis to be performed in the context of the Generalised Beam Theory (GBT). The novelty relies in formulating the problem in the spirit of Kantorovich's semi variational method, namely using the dynamic modes of an unconstrained planar frame as in plane deformation modes. Warping is then evaluated from the post processing of these in plane modes, thus reversing the strategy of the classical GBT procedure. The new procedure does not require several steps of the classical algorithm for the determination of the conventional modes, in which bending, shear and local modes are evaluated separately, and is applicable indifferently to open, partially closed and closed sections. The efficiency and ease of use of the method are outlined by means of two examples, aimed to describe the linear elastic behaviour of thin walled members.
\end{abstract}

\section{Introduction}

The Generalised Beam Theory (GBT) is a powerful tool for the elastic and buckling analysis of thin walled members (TWM). The basic ideas of the method have been originally proposed by Schardt $[1,2]$ and disseminated in English by Davies and co workers [3 7] while its large diffusion in the scientific commu nity is due to the strong impulse given to it in the last decade by Camotim and co workers [ 8 27]. They generalised the method to include new aspects, not present in the original formulation, and combined it with a FE approach [8 10], so that GBT is now applicable to anisotropic members [11], branched open sections [12], closed or partially closed sections [13], circular sections [14], non standard support conditions [15,16], frames of TWM [17], buckling problems $[10,13,14,1822]$, linear dynamic problems $[23,24]$, and post buckling problems [25 27]. In recent years, Adany and Schafer $[28,29]$ and Casafront et al. [30] applied the principles at the basis of the GBT to reduce the number of freedoms required in performing buckling investigations using the finite strip method and finite element method, respectively.

As well known, GBT considers a TWM as an assembly of (generally, but not necessarily, flat) thin plates, free to bend in the plane orthogonal to the member axis. Thus, GBT accounts for deformable cross sections, differently from the classical Vlasov theory [31] in which the cross section keeps its original shape. The basic idea of the method consists in describing the displace ment field of the TWM as a linear combination of assumed

\footnotetext{
* Corresponding author. Tel.: +6129351 5215; fax: +61293513343.

E-mail address: gianluca.ranzi@sydney.edu.au (G. Ranzi).
}

'deformation modes' of the cross section (including in plane and warping components), and 'amplitude modes', which are unknown functions depending on the axial coordinate. A varia tional principle, as the virtual work equation, provides the weak formulation of the problem, leading to a system of ordinary differential equations in the unknown amplitudes, with the relevant boundary conditions. These equations, equal in number to the deformation modes considered, generalise the classical Vlasov beam theory, where the latter can be described using four amplitude functions, each associated to a rigid motion of the section, namely three translations (two flexures and an extension) and one rotation (torsion) around the shear centre. Even if not initially noted by Schardt, this approach falls within Kantorovich's semi variational method, aimed at reducing the dimensionality of a problem through a technique of partially assumed modes. Thus, in the case of the GBT, a three dimensional continuous problem is transformed into a vector valued one dimensional problem. In particular, the GBT method consists of two phases: (1) the choice of the deformation modes, referred to as 'cross sectional analysis', and (2) the solution of the amplitude equations, denoted as 'member analysis'. The fundamental step of this method relies on its ability to determine a suitable set of deformation modes.

A brief overview of the essence of the classical GBT is provided in the following, partly for readers not fully familiar with this approach, to highlight the procedure involved in the evaluation of the conven tional deformation modes and to better outline the contribution of the proposed work. Throughout this paper and consistently with the definitions provided in [8], conventional modes are assumed to include the rigid body modes, the distortional ones, the local (bending) ones complemented, when dealing with closed sections, with a shear mode to depict the case of pure torsional shear flow. 
The first step of the GBT formulation is to discretise the cross section of a member in plate segments (with nodes inserted at their ends) along which linearly varying warping functions are assumed to exist. By following the methodology commonly used with the direct stiffness method, unit warping displacements are applied to one node at the time, while keeping all the remaining ones equal to zero, thus creating a set of 'warping modes'. The corresponding in plane tangential displacements of the segments are determined for each of these modes based on the zero shear Vlasov condition (valid for open cross sections). This procedure leads to a loss of compatibility at the 'natural nodes' (i.e. at the corners of the plates). A rigid body kinematic problem is then solved to restore the compatibility of the translations by applying normal displacements and rotations to each plate segment. After this, the force method is used to restore compatibility of the rotations at the natural nodes, while treating the plate segments as (deformable) continuous members and assum ing their corners restrained by pinned supports. With this procedure, a basis of linearly independent modes (with number equal to the number of nodes of the section) is created. This set of 'warping modes', however, is not exhaustive, since it is associated to deforma tions in which the nodes translate in the cross section plane. 'Bending modes', instead, in which the plate deforms by leaving the corners (practically) immovable and without warping, cannot be determined with this procedure. The way that GBT addresses this problem is to restart a new calculation in which unit displacements are assigned, this time normal to the plate segments, at 'non natural' intermediate nodes (i.e. not at the corners), followed, also in this instance, by the solution of the elastic problem of a continuous member. This second set of modes, however, does not include deformations of closed sections in which the displacements are essentially tangential, triggering shear deformations of the plates, and therefore referred to as 'shear modes'. To determine these latter ones another ad hoc procedure needs to be applied, which induces unit tangential displacements to each segment of the cross section belonging to closed cells (based on the considerations that membrane shear strains are negligible in segments included in open branches).

The different deformation modes constructed, based on the procedures previously outlined, are local type in nature because they involve nontrivial displacements only in a few adjacent segments. Remaining consistent with their derivations, these modes could be used in this form, in a similar manner as, for example, the finite strip approach is applied to TWM analysis or splines are used to describe extended functions (e.g., [32 36]). Such a local type representation, however, is not convenient, if one desires to use few significant modes to capture the main structural behaviour. Therefore, a change of basis is then per formed in the classical GBT to obtain global type deformation modes. This is obtained as the eigenvectors of a properly chosen eigenvalue problem, able to simplify the amplitude equations.
It is in authors' opinion that an unified procedure for the determination of the conventional deformation modes would con tribute to a wider diffusion and use of the GBT approach. In this context, a new version of the method is proposed here, which, in the spirit of the semi variational method selects the conventional deformation modes directly defined on the whole domain and chosen as the eigenvectors of a positive semi definite eigenvalue problem. The free dynamics of the unconstrained planar frame, represented by the plate segments forming the cross section placed at their mid lines, is chosen as the eigenvalue problem. Since the frame is free in its plane, it possesses rigid motions that account for the Vlasov beam theory, and flexural modes which account for deformation modes. Once the planar modes are determined, e.g. using a standard finite element analysis, even performed with a commercial software, the cross sectional analysis is completed by evaluating the corresponding warping displacements based on conditions enforced on the shear strain. Among these, the purely extensional mode appears as an arbitrary quantity rising from integration. In this way, the strategy used by the classical theory is reversed, in the sense that in plane components are evaluated first, and warping components successively.

This paper starts by briefly recalling the basis of the GBT, limiting the description to the first order analysis. This is fol lowed by the new proposed cross sectional analysis and its ease of use is outlined by means of two applications on simply supported TWM. For clarity, the procedure proposed for the calculation of the warping displacements, starting from the in plane ones, is detailed with an example in Appendix A.

\section{Basis of the GBT approach}

A generic thin walled member is considered with (a) open, (b) closed or (c) partially closed cross section formed with flat plates (Fig. 1). The displacement field $\mathbf{u}(s, z)$ of an arbitrary point $\mathrm{P}(s, z)$ lying in the mid plane of the section thickness is expressed as (Fig. 2):

$\mathbf{u}(s, z)=u(s, z) \mathbf{e}_{s}(s)+v(s, z) \mathbf{e}_{y}(s)+w(s, z) \mathbf{e}_{z}(s)$

where $s$ is the curvilinear abscissa (if necessary defined on several branches) along the section mid line $\mathcal{C}, z$ is the coordinate along the member axis, $\mathbf{e}_{s}(s), \mathbf{e}_{y}(s)$ and $\mathbf{e}_{z}(s)$ are unit vectors in the tangential, normal and bi normal directions at the abscissa $s$, respectively, and $u(s, z), v(s, z)$ and $w(s, z)$ are the displacement components in the same triad.

\subsection{Displacement and strain fields}

In the framework of the GBT, and making use of Kantorovich's semi variational method, the displacement components of points
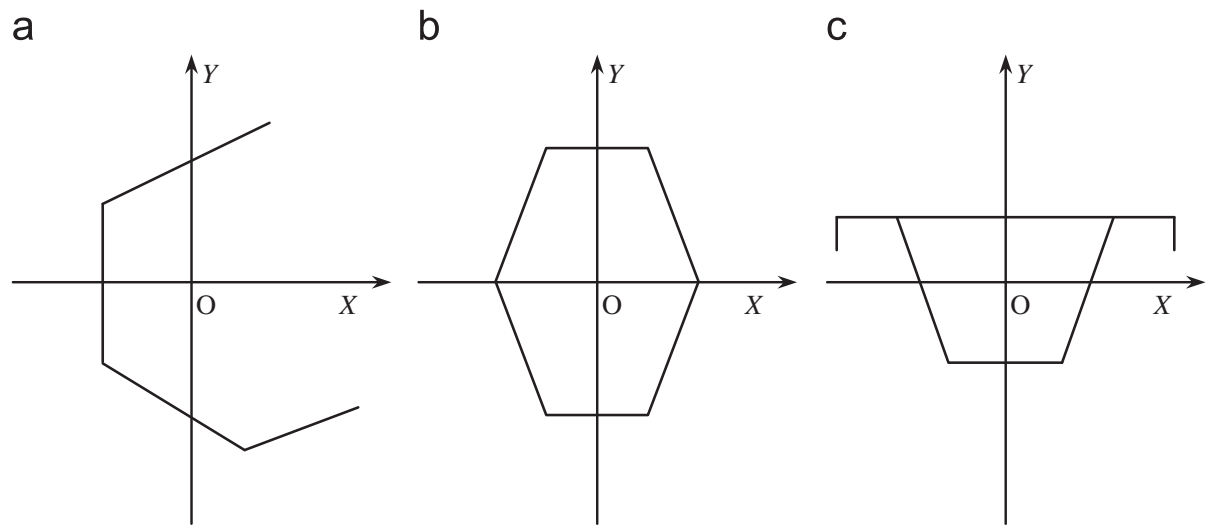

Fig. 1. Generic thin-walled cross-sections: (a) open cross-section; (b) closed cross-section; and (c) partially-closed cross-section. 


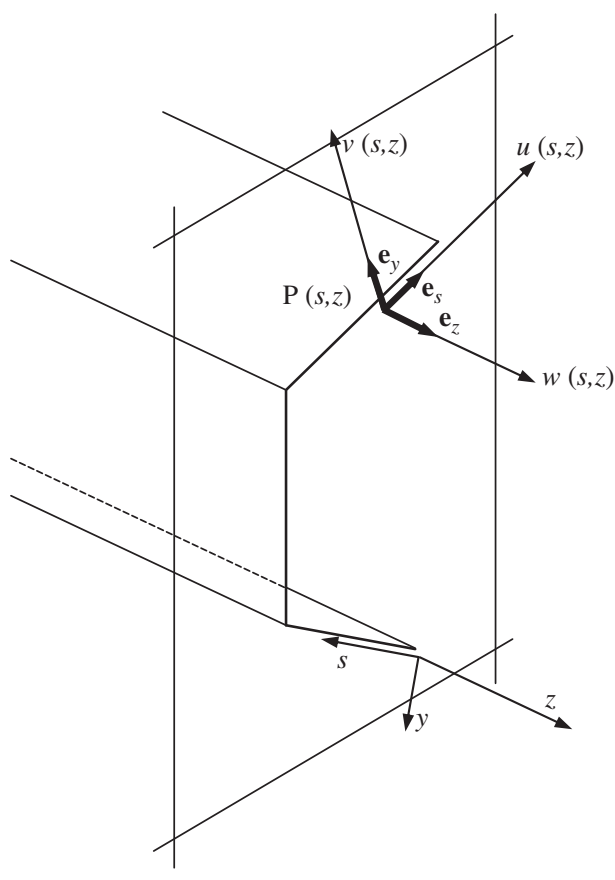

Fig. 2. Displacement field.

$\mathrm{P}(s, 0, z)$ lying on the mid surface $y=0$ are expressed as a linear combination of $K$ assumed deformation modes, depending on the abscissa $s$, and unknown amplitude functions, depending on the coordinate $z$, namely:

$u(s, z)=\sum_{k}^{K} U_{k}(s) \varphi_{k}(z)$

$v(s, z)=\sum_{k}^{K} V_{k}(s) \varphi_{k}(z)$

$w(s, z)=\sum_{k}^{K} W_{k}(s) \varphi_{k, z}(z)$

where $U_{k}(s), V_{k}(s)$ and $W_{k}(s)$ are the three components of the $k$ th deformation mode, $\varphi_{k}(z)$ describes the amplitude of the $k$ th mode along the member axis and a comma denotes differentia tion with respect the variable that follows. The displacement $\mathbf{d}(s, y, z)=d_{s}(s, z) \mathbf{e}_{s}(s)+d_{y}(s, z) \mathbf{e}_{y}(s)+d_{z}(s, z) \mathbf{e}_{z}(s)$ of an arbitrary point $\mathrm{Q}(s, y, z)$ located within the thickness of the plate segments is evaluated according to the Kirchhoff plate model, namely:

$d_{s}(s, y, z)=u(s, z) \quad y v_{, s}(s, z)$

$d_{y}(s, y, z)=v(s, z)$

$d_{z}(s, y, z)=w(s, z) \quad y v_{, z}(s, z)$

or, based on Eq. (2):

$\mathbf{d}(s, y, z)=\left[\begin{array}{cc}\sum_{k}^{K}\left[U_{k}(s)\right. & \left.y V_{k, s}(s)\right] \varphi_{k}(z) \\ & \sum_{k}^{K} V_{k}(s) \varphi_{k}(z) \\ \sum_{k}^{K}\left[\begin{array}{ll}W_{k}(s) & y V_{k}(s)\end{array}\right] \varphi_{k, z}(z)\end{array}\right]$

The corresponding infinitesimal strain fields $\varepsilon_{s}=d_{s, s}, \varepsilon_{z}=d_{z, z}$, $\gamma_{z s}=d_{z, s}+d_{s, z}$ are made of two contributions, a membrane strain component $\boldsymbol{\varepsilon}^{M}=\left(\varepsilon_{s}^{M}, \varepsilon_{z}^{M}, \gamma_{z s}^{M}\right)^{T}$, relevant to the $y=0$ plane, and a flexural strain component $\boldsymbol{\varepsilon}^{F}=\left(\varepsilon_{s}^{F}, \varepsilon_{z}^{F}, \gamma_{z s}^{F}\right)^{T}$, proportional to the $y$ coordinate. Based on Eqs. (2) and (4), the six component strain field $\boldsymbol{\varepsilon}=\left(\varepsilon_{s}^{M}, \varepsilon_{z}^{M}, \gamma_{z s}^{M} ; \varepsilon_{s}^{F}, \varepsilon_{z}^{F}, \gamma_{z s}^{F}\right)^{T}$ can be written as:

$$
\boldsymbol{\varepsilon}=\left[\begin{array}{c}
u_{, s}(s, z) \\
w_{, z}(s, z) \\
u_{, z}(s, z)+w_{, s}(s, z) \\
y v_{, s s}(s, z) \\
y v_{, z z}(s, z) \\
2 y v_{, s z}(s, z)
\end{array}\right]=\left[\begin{array}{c}
\sum_{k}^{K} U_{k, s}(s) \varphi_{k}(z) \\
\sum_{k}^{K} W_{k}(s) \varphi_{k, z z}(z) \\
\sum_{k}^{K}\left[U_{k}(s)+W_{k, s}(s)\right] \varphi_{k, z}(z) \\
y \sum_{k}^{K} V_{k, s s}(s) \varphi_{k}(z) \\
y \sum_{k}^{K} V_{k}(s) \varphi_{k, z z}(z) \\
2 y \sum_{k}^{K} V_{k, s}(s) \varphi_{k, z}(z)
\end{array}\right]
$$

According to the two fundamental Vlasov's hypotheses, for mulated for open cross sections, the plate components are assumed: (i) inextensible in the tangential direction, i.e. $\varepsilon_{s}^{M}=0$, and $\left(\mathrm{ii}_{1}\right)$ shear indeformable in their middle plane, i.e. $\gamma_{z s}^{M}=0$. This latter condition, however, is relaxed for closed sections (or, more in general, for plates belonging to closed loops of partially closed cross sections), and substituted by $\left(\mathrm{ii}_{2}\right) \gamma_{z s}^{M}=$ const stepwise with respect to $s$ (i.e. on each element of a closed loop), according to the Bredt theory for torsion. As a consequence of $\left(\mathrm{ii}_{1}\right)$ and $\left(\mathrm{ii}_{2}\right)$, no shear strains due to shear forces (as for example predicted by the Jourawsky theory) are permitted on any kind of section. It is assumed here that the deformation modes appearing in Eqs. (2) are selected in such a way to identically satisfy the constraints (i) and ( $\left.\mathrm{ii}_{1}\right)$ or, if appropriate, $\left(\mathrm{ii}_{2}\right)$.

\subsection{Stress field}

Due to the internal constrains, the associate stress field $\boldsymbol{\sigma}=\left(\sigma_{s}^{M}, \sigma_{z}^{M}, \tau_{z s}^{M} ; \sigma_{s}^{F}, \sigma_{z}^{F}, \tau_{z s}^{F}\right)^{T}$ is made of an active part $\boldsymbol{\sigma}_{a}$ and a reactive one $\boldsymbol{\sigma}_{r}$, i.e. $\boldsymbol{\sigma}=\boldsymbol{\sigma}_{a}+\boldsymbol{\sigma}_{r}$, in which $\boldsymbol{\sigma}_{a}$ is linked to the non zero strains, and $\boldsymbol{\sigma}_{r}$ collects the stresses which spend zero work on the strain field, namely $\boldsymbol{\sigma}_{r}^{T} \boldsymbol{\varepsilon}=0$. Accordingly, these can be expressed as $\boldsymbol{\sigma}_{a}=\left(0, \sigma_{z}^{M}, 0 ; \sigma_{s}^{F}, \sigma_{z}^{F}, \tau_{z s}^{F}\right)^{T}$ on plates lying on open branches and $\boldsymbol{\sigma}_{a}=\left(0, \sigma_{z}^{M}, \tau_{z s}^{M} ; \sigma_{s}^{F}, \sigma_{z}^{F}, \tau_{z s}^{F}\right)^{T}$ on plates on closed branches, while $\boldsymbol{\sigma}_{r}=\left(\sigma_{s}^{M}, 0, \tau_{z s}^{M} ; 0,0,0\right)^{T}$ on all plates. It should be noted that $\tau_{z s}^{M}$ is made of two contributions: an active one due to twist of closed branches and a reactive one due to shear forces applied to any kind of section. Assuming linear (plane stress) elastic material properties, the constitutive relationship can be expressed as:

$\boldsymbol{\sigma}=\mathbf{E} \boldsymbol{\varepsilon}+\boldsymbol{\sigma}_{r}$

where:

$\mathbf{E}=\left[\begin{array}{cccccc}0 & 0 & 0 & 0 & 0 & 0 \\ 0 & E & 0 & 0 & 0 & 0 \\ 0 & 0 & G & 0 & 0 & 0 \\ 0 & 0 & 0 & \frac{E}{1-v^{2}} & \frac{v E}{1-v^{2}} & 0 \\ 0 & 0 & 0 & \frac{v E}{1-v^{2}} & \frac{E}{1-v^{2}} & 0 \\ 0 & 0 & 0 & 0 & 0 & G\end{array}\right]$

is the elastic matrix, containing elastic moduli $E$ and $G$, in which, as usually accepted, the Poisson ratio $v$ has been ignored in the entry $(2,2)$.

Evaluation of the reactive part of stress is beyond the scope of this paper, consistently with the displacement method followed. Hence, focusing the attention on the active part only, and using 
Eq. (2) in the constitutive law of Eq. (6), it follows that:

$\sigma_{z}^{M}(s, z)=\sum_{k}^{K} E W_{k}(s) \varphi_{k, z z}(z)$

$\tau_{z s}^{M}(s, z)=\sum_{k}^{K} G\left[U_{k}(s)+W_{k, s}(s)\right] \varphi_{k, z}(z)$

$\sigma_{s}^{F}(s, y, z)=y \sum_{k}^{K} \frac{E}{1} v^{2}\left[V_{k, s s}(s) \varphi_{k}(z)+v V_{k}(s) \varphi_{k, z z}(z)\right]$

$\sigma_{z}^{F}(s, y, z)=y \sum_{k}^{K} \frac{E}{1} v^{2}\left[V_{k}(s) \varphi_{k, z z}(z)+v V_{k, s s}(s) \varphi_{k}(z)\right]$

$\tau_{z s}^{F}(s, y, z)=2 y \sum_{k}^{K}\left[G V_{k, s}(s) \varphi_{k, z}(z)\right]$

It should be noted that, due to the Poisson effect, the normal flexural stresses are the superposition of two contributions, whose relative amplitudes at the abscissa $z$ depends on the magnitudes of $\varphi_{k}(z)$ and $\varphi_{k, z z}(z)$; consequently, the stress distri bution changes (in shape) with $z$. In the particular case in which $\varphi_{k}(z)$ is harmonic, the stress variation is independent of $z$ and its amplitude varies along the member length.

\subsection{Weak form of the equilibrium}

The weak form of the problem is derived by means of the principle of virtual work, which is expressed as:

$\int_{V} \boldsymbol{\sigma}^{T} \delta \boldsymbol{\varepsilon} d V=\int_{S} \mathbf{f}^{T} \delta \mathbf{u} d S$

where $\mathbf{f}(s, z)=f_{s}(s, z) \mathbf{e}_{s}(s)+f_{y}(s, z) \mathbf{e}_{y}(s)+f_{z}(s, z) \mathbf{e}_{z}(s)$ represents the surface loads, applied at the mid surface of the member, and the $\delta$ denotes a virtual quantity. Performing standard steps of variational calculus, the following system of ordinary differential equations in the unknown amplitude functions is obtained:

$\mathbf{C} \varphi_{, z z z z}+\mathbf{D} \varphi_{, z z}+\mathbf{B} \varphi=\mathbf{p}$

and the relevant boundary conditions at $z=0, L$ :

$$
\left(\mathbf{C} \boldsymbol{\varphi}_{, z z}+\mathbf{D}^{a} \boldsymbol{\varphi}\right) \delta \boldsymbol{\varphi}_{, z}=\mathbf{0}
$$

$\left[\mathbf{C} \boldsymbol{\varphi}_{, z z z}+\left(\begin{array}{lll}\mathbf{D}^{a} & \mathbf{D}^{c}\end{array}\right) \boldsymbol{\varphi}_{, z} \quad \mathbf{p}^{z}\right] \delta \boldsymbol{\varphi}=\mathbf{0}$

In the previous equations $\mathbf{C}, \mathbf{D}^{a}, \mathbf{D}^{b}, \mathbf{D}^{c}$ and $\mathbf{B}$ are $K \times K$ symmetric stiffness matrices with coefficients:

$C_{h k}=\int_{\mathcal{C}}\left(E t W_{h} W_{k}+\frac{E t^{3}}{12\left(1 v^{2}\right)} V_{h} V_{k}\right) d s$

$D_{h k}^{a}=\int_{\mathcal{C}}\left(\frac{v E t^{3}}{12\left(1 v^{2}\right)} V_{h} V_{k, s s}\right) d s$

$D_{h k}^{b}=\int_{\mathcal{C}}\left(\frac{v E t^{3}}{12\left(1 \quad v^{2}\right)} V_{h, s s} V_{k}\right) d s$

$D_{h k}^{c}=\int_{\mathcal{C}}\left(G t\left(U_{h}+W_{h, s}\right)\left(U_{k}+W_{k, s}\right)+\frac{G t^{3}}{3} V_{h, s} V_{k, s}\right) d s$

$D_{h k}=D_{h k}^{a}+D_{h k}^{b} \quad D_{h k}^{c}$

$B_{h k}=\int_{\mathcal{C}}\left(\frac{E t^{3}}{12\left(1 v^{2}\right)} V_{h, s s} V_{k, s s}\right) d s$ in which $\mathbf{p}$ and $\mathbf{p}^{z}$ are load $K$ vectors, whose coefficients are defined as:

$p_{h}=\int_{\mathcal{C}} f_{s} U_{h} d s+\int_{\mathcal{C}} f_{y} V_{h} d s \int_{\mathcal{C}} f_{z, z} W_{h} d s ; \quad p_{h}^{z}=\int_{\mathcal{C}} f_{z} W_{h} d s$

In Eq. (11), matrix $\mathbf{C}$ accounts for internal work spent by stresses $\sigma_{z}^{M}, \sigma_{z}^{F}$ in the dual strains; matrices $\mathbf{D}^{a}$ and $\mathbf{D}^{b}$ include the work spent by flexural stresses due to the Poisson effect; matrix $\mathbf{D}^{c}$ depicts the work of tangential stresses due to the Bredt torsion (i.e. relevant to closed branches) and De Saint Venant torsion (i.e. relevant to open branches), respectively; finally, matrix $\mathbf{B}$ is associated with transversal bending of the plates in the cross section plane. It is worth noting that in Vlasov theory $D_{h k}^{a}=D_{h k}^{b}=$ $B_{h k}=0$ and the second contribution in $C_{h k}$ (related to bending of plates in the longitudinal direction) is not accounted for.

\section{Cross-sectional analysis}

The fundamental task to be performed for an adequate modelling consists of the identification of suitable deformation modes (described by $U_{k}(s), V_{k}(s)$ and $W_{k}(s)$ in Eq. (2)) capable of describing the loss of initial shape of the cross section. Compared with the classical theory, discussed in Section 1, a new approach is presented in the following to achieve this, in which the deformation modes are taken as the dynamic eigenmodes of the cross section, considered as a free planar frame.

\subsection{Discrete models for cross section analysis}

To perform the dynamic analysis of the cross section, a discrete model is adopted and implemented using the finite element procedure. This leads to the algebraic eigenvalue problem:

(K $\lambda \mathbf{M}) \mathbf{q}=\mathbf{0}$

where $\mathbf{K}$ is the stiffness matrix, $\mathbf{M}$ the mass matrix and $\mathbf{q}$ is the vector of the nodal displacements of the section. Three different one dimensional elements are considered, consisting of (Fig. 3): (i) a 6 DOF finite element with cubic and linear polynomials for the transverse and axial displacements, respectively, and masses equally lumped at its end nodes (referred to as FE 1 in the following); (ii) a 6 DOF finite element with same polynomial interpolation functions of FE 1 but with consistent masses (referred to as FE 2); and (iii) a 8 DOF stiffness element same as FE 2 with the order of the polynomial approximating the transverse displacement increased to five (referred to as FE 3). These elements are derived based on standard finite element procedures (e.g., [37 39]).

\subsection{Inextensibility conditions}

Solving the eigenvalue problem in the form specified in Eq. (14) produces the eigenmodes of the section whose members are free to stretch. However, to enforce inextensibility, internal constraints need to be introduced in the model. This is carried out following available procedures commonly used in finite element modelling (e.g., [37]), also applied to thin walled structures in recent years (e.g., $[30,40]$ ).

Referring to the generic member $e$, connecting nodes $i$ and $j$, whose axis forms an angle $\alpha_{e}$ with the $X$ axis, these (linearised) constraints can be expressed in local coordinates as $u_{1}^{e} u_{2}^{e}=0$ (Fig. 3) or in global coordinates as:

$\left(\begin{array}{ll}u_{j} & u_{i}\end{array}\right) \cos \alpha_{e}+\left(\begin{array}{ll}v_{j} & v_{i}\end{array}\right) \sin \alpha_{e}=0 \quad e=1,2, \ldots, M$

or, in matrix form:

$\mathbf{A q}=\mathbf{0}$

where $\mathbf{A}$ is a $M \times N$ matrix, $M$ being the number of elements and $N>M$ the number of dofs; and $\mathbf{q}=\left\{\ldots, u_{j}, v_{j}, \theta_{j}, \ldots\right\}^{T}$ collects the $N$ 

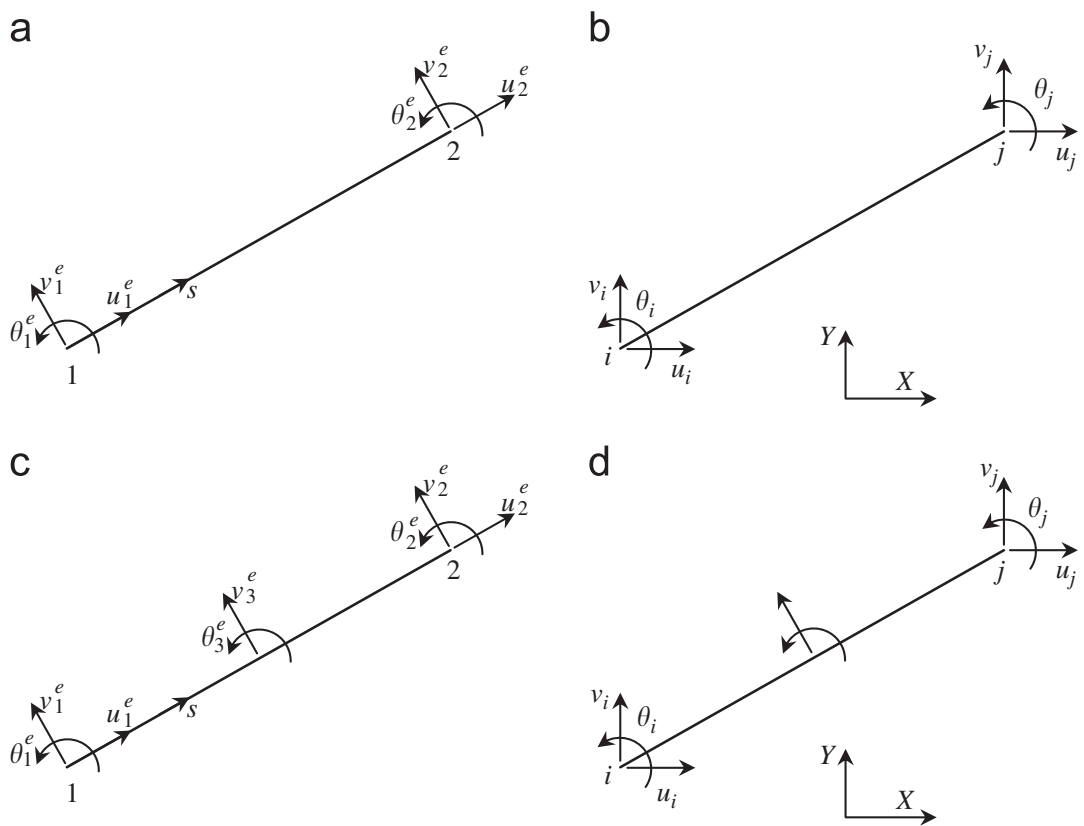

Fig. 3. Nodal displacements in local and global coordinates for 6 DOF and 8 DOF finite elements: (a) nodal displacements in local coordinates (6 DOF finite element); (b) nodal displacements in global coordinates (6 DOF finite element); (c) nodal displacements in local coordinates (8 DOF finite element); and (d) nodal displacements in global coordinates (8 DOF finite element).

nodal displacements defined in the global reference system $(X, Y)$. The previous equation permits to express the $M$ slave dofs $\mathbf{q}_{\mathrm{s}}$ in terms of the $N$ M master dofs $\mathbf{q}_{M}$. Partitioning Eq. (15b) as:

$\left[\begin{array}{ll}\mathbf{A}_{M} & \mathbf{A}_{S}\end{array}\right]\left[\begin{array}{l}\mathbf{q}_{M} \\ \mathbf{q}_{S}\end{array}\right]=\mathbf{0}$

where $\mathbf{A}_{S}$ is a non singular $M \times M$ sub matrix, the slave dofs included in $\mathbf{q}_{S}$ can be expressed as a function of the master dofs $\mathbf{q}_{M}$ based on:

$\mathbf{q}=\mathbf{R} \mathbf{q}_{M}$

where:

$\mathbf{q}=\left[\begin{array}{l}\mathbf{q}_{M} \\ \mathbf{q}_{S}\end{array}\right] ; \quad \mathbf{R}=\left[\begin{array}{c}\mathbf{I} \\ \mathbf{A}_{S}^{-1} \mathbf{A}_{M}\end{array}\right]$

Requiring the virtual work included in equation $\delta \mathbf{q}^{T}\left(\begin{array}{ll}\mathbf{K} & \lambda \mathbf{M}\end{array}\right)$ $\mathbf{q}=0$ to be nil for any $\delta \mathbf{q}$ satisfying the constraint enforced with Eq. (17), i.e. $\delta \mathbf{q}=\mathbf{R} \delta \mathbf{q}_{M}$, the algebraic eigenvalue problem for the internally restrained cross section becomes:

$\left(\begin{array}{ll}\mathbf{K}_{R} & \lambda \mathbf{M}_{R}\end{array}\right) \mathbf{q}_{M}=0$

where the two reduced order $\left(\begin{array}{lll}N & M\end{array}\right) \times\left(\begin{array}{ll}N & M\end{array}\right)$ matrices $\mathbf{K}_{R}$ and $\mathbf{M}_{R}$ are defined as:

$\mathbf{K}_{R}=\mathbf{R}^{T} \mathbf{K R} ; \quad \mathbf{M}_{R}=\mathbf{R}^{T} \mathbf{M R}$

\subsection{Dynamic eigenmodes and warping functions}

The in plane deformation modes $U_{k}(s)$ and $V_{k}(s)$ appearing in Eq. (2) are obtained by solving the eigenvalue problem in Eq. (19). In this case, the eigenvalues $\lambda$ represent the squared eigenfrequencies, and the associated eigenvectors $\mathbf{q}_{M}$ (suitably normalised by taking, for example, $\max \left|\mathbf{q}_{M}\right|=1$ ) are nodal displacements representing, based on the adopted interpolation functions, the deformation modes in the plane of the cross section. Due to the inextensibility condition, it follows that $U_{k}(s)$ is piecewise constant on $s$, i.e. $U_{k}(s)=U_{k}^{e}(e=1,2, \ldots, M)$. Since the frame is not restrained (i.e. it is free in its plane), a triple eigenvalue $\lambda=0$ is found, associated to three rigid modes of the section. These are taken as the two translations and the rotation around an arbitrary selected point (e.g., but not necessarily, the shear centre of the Vlasov theory).

According to the suggested procedure, the in plane deformation modes (excluding the rigid ones) are the infinite deflections $\mathbf{u}(\mathrm{s})$ assumed to occur at the cross section when loaded by (active) distributed forces $\mathbf{f}(\mathrm{s})$ proportional to the displacements themselves (indeed, $\mathbf{f}=m \mathbf{u}=m \omega^{2} \mathbf{u}$, with $\omega$ being the circular frequency and $m$ is the distributed mass per unit length). Consequently, since $\mathbf{f}(s)$ are self equilibrated forces due to the absence of constraint reac tions, the deformation modes have zero mean value. Because of this, displacements are usually observed to occur at the corners of the cross section in the calculated deformation modes. The latter represents a peculiarity of the proposed class of deformation modes, if compared with those derived with the classical GBT.

The warping deformations undergone by the member in each mode (including the rigid ones) are successively obtained from the nil $\left(\mathrm{ii}_{1}\right)$, or constant $\left(\mathrm{ii}_{2}\right)$, membrane skear assumption. Recalling that (Eq. (5)) $\gamma_{z s}^{M}=u_{, z}+w_{, s}=\sum_{k}\left[U_{k}(s)+W_{k, s}(s)\right] \varphi_{k, z}(z)$,
it follows that:

$\gamma_{z s}^{M}=\sum_{k}^{K} \Gamma_{k}(s) \varphi_{k, z}(z)$

with:

$\Gamma_{k}(s)=U_{k}(s)+W_{k, s}(s)$

and $\Gamma_{k}(s)$ results in a piecewise constant function, i.e. $\Gamma_{k}(s)=\Gamma_{k}^{e}$ $(e=1,2, \ldots, M)$, which equals zero for elements in open branches or unknown values for those included in closed branches. The linear warping along each plate element can be expressed as a function of its values at its end nodes as:

$W_{k}(s)=W_{k, s}(s) s+W_{k}^{i_{1}}=\frac{W_{k}^{i_{2}} \quad W_{k}^{i_{1}}}{b^{e}} s+W_{k}^{i_{1}} \quad e=1,2, \ldots, M$

and $i_{1}, i_{2} \in \mathcal{I}_{\text {nodes }}^{e}$

where $W_{k}^{i_{1}}$ and $W_{k}^{i_{2}}$ depict the values of warping at the first node $i_{1}$ (i.e. at $s=0$ ) and the end one $i_{2}$ (i.e. at $s=b^{e}$ ), respectively, of element $e$ of length $b^{e}$ based on the positive direction specified by an arbitrary location vector; $i_{1}$ and $i_{2}$ are integers included in the set 
$\mathcal{I}_{\text {nodes }}^{e}$. Based on this, Eq. (21b) can be rewritten for each element forming the cross section in terms of the node warping as:

$\Gamma_{k}^{e}=U_{k}^{e}+\frac{W_{k}^{i_{2}} W_{k}^{i_{1}}}{b^{e}} \quad e=1,2, \ldots, M \quad$ and $\quad i_{1}, i_{2} \in \mathcal{I}_{\text {nodes }}^{e}$

In the case of open sections (i.e. without closed loops) the warping functions are defined once the $n$ unknown values $W_{k}^{i}$, which depict the warping at the $i$ th node (with $i=1, \ldots, n$ ), are determined. This is achieved by enforcing for each member:

$\Gamma_{k}^{e}=U_{k}^{e}+\frac{W_{k}^{i_{2}} W_{k}^{i_{1}}}{b^{e}}=0$

Since one of these unknowns remains arbitrary, it describes the uniform extension of the member. To make the warping orthogonal to the extension, it is convenient to choose the remaining arbitrary constant in such a way that the average $k$ mode warping is zero, i.e.:

$\int_{C} W_{k}(s) \mathrm{d} s=0$

In the case of closed cross section, or those containing closed loops, the constants $\Gamma_{k}^{e}$ are not equal to zero, but are related, via the constitutive law, to the (unknown) tangential stress flow $Q_{k}^{j}$ acting on the $j$ th loop $C_{j}$. For an element $e$ belonging to this loop Eq. (24) must then be replaced by:

$G t_{e} \Gamma_{k}^{e}=G t_{e}\left(U_{k}^{e}+\frac{W_{1, k}^{e} W_{0, k}^{e}}{b^{e}}\right)=\sum_{j \in \mathcal{I}_{c}^{e}}( \pm) Q_{k}^{j}$

where the sum is extended to the set $\mathcal{I}_{C}^{e}$ of all integers $j$ labelling the closed loops $C_{j}$ to which member $e$ belongs to. The sign of the positive flow is specified by the arbitrary positive rotation assigned to each closed loop. A positive (negative) sign is then used in the summation for $Q_{k}^{j}$ in the right hand side of Eq. (26) when the directions of the adopted element location vector and of the shear flow passing through the $e$ element coincide (differ). The procedure required for the calculation of the warping modes is illustrated by an example in Appendix A.

The determination of the warping shapes concludes the evalua tion of the deformation modes, so that the stiffness matrices (Eqs. 12) and the load vector (Eq. 13) can finally be evaluated.

\section{Member linear analysis}

The member analysis requires solving the set of ordinary differential equations (Eq. (10)). In order to highlight the ability of the proposed deformation modes to capture the behaviour of
TWM, the simplest boundary conditions are considered for the member analysis, i.e. a simply supported member torsionally restrained and free to warp at the ends (i.e. $u(s, \bar{z})=v(s, \bar{z})=0$ and $w_{, z}(s, \bar{z})=0$ at $\left.\bar{z}=0, L\right)$. The forces $\mathbf{f}$ are assumed harmonically varying with $z$, namely:

$f_{s}=f_{s n} \sin \left(\frac{n \pi z}{L}\right) ; \quad f_{y}=f_{y n} \sin \left(\frac{n \pi z}{L}\right) ; \quad f_{z}=f_{z n} \cos \left(\frac{n \pi z}{L}\right)$

$(27 a-c)$

so that the load vector in Eq. (10) is $p_{h}=p_{h}^{0} \sin (n \pi z / L)$, with $n$ being an integer. Such a problem is well suited for the following amplitude functions:

$\varphi_{k}=a_{k} \sin \left(\frac{n \pi z}{L}\right), \quad k=1,2, \ldots, K$

where $a_{k}$ are unknown constants. Eq. (10) is thus transformed in an algebraic problem for $a_{k}$, reading:

$\sum_{k}^{K}\left[C_{h k}\left(\frac{n \pi}{L}\right)^{4} \quad D_{h k}\left(\frac{n \pi}{L}\right)^{2}+B_{h k}\right] a_{k}=p_{h}^{0}, \quad h=1,2, \ldots, K$

Once Eq. (29) is solved, the displacement field can be calculated using Eqs. (2) and (4) and, recalling the introduced constitutive relationships of Eq. (6), the active stresses can be obtained, based on Eqs. (8) and (9):

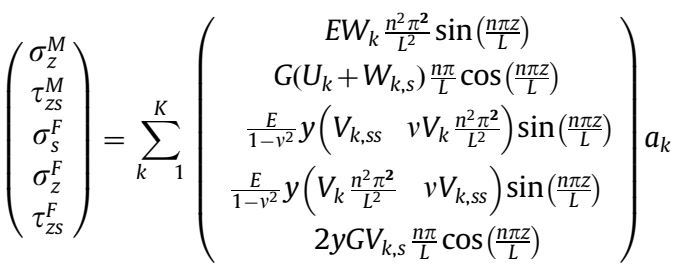

\section{Numerical results}

The proposed approach is illustrated in the following consid ering a lipped section with a distributed loading applied to the web and to the top flange, respectively (Fig. 4). For clarity, these loading scenario have been referred to as Loading Cases 1 and 2 . The deformation modes obtained by the cross sectional analysis are presented in Fig. 5 where, for each mode, the displacements taking place in the plane of the cross section have been labelled as ' $u v$ ' and their corresponding warping distributions as ' $w$ '. For illustrative purposes the first 19 modes have been provided.

Considering a $1000 \mathrm{~mm}$ long member, a plate thickness of $1 \mathrm{~mm}$ and a sinusoidal load (with wave number $n=1$ ) of amplitude $1 \mathrm{~N} / \mathrm{mm}$, the results of the linear elastic analysis, calculated using

b

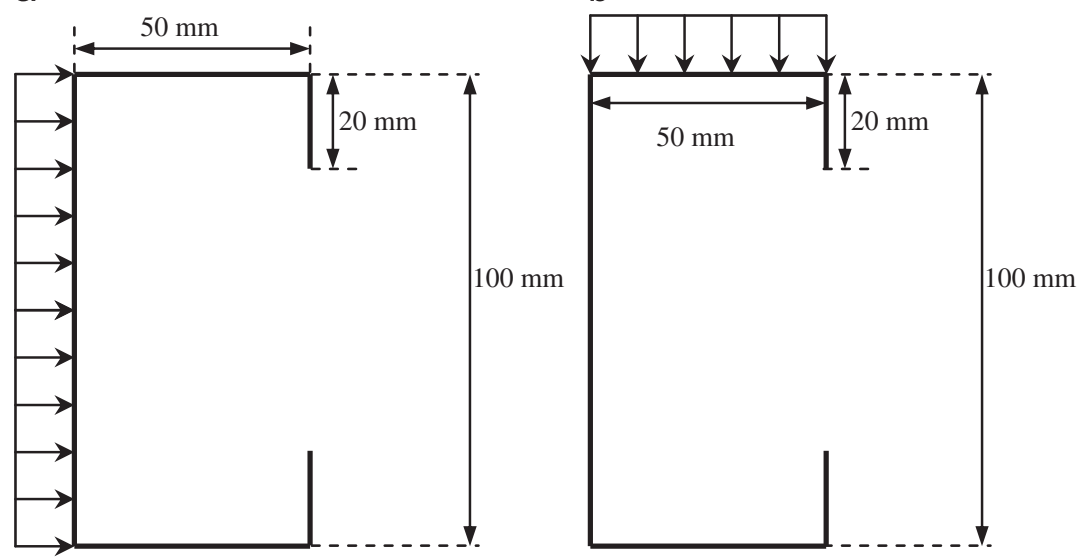

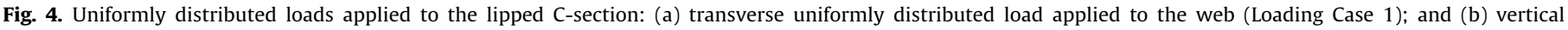
uniformly distributed load applied to the top flange (Loading Case 2). 

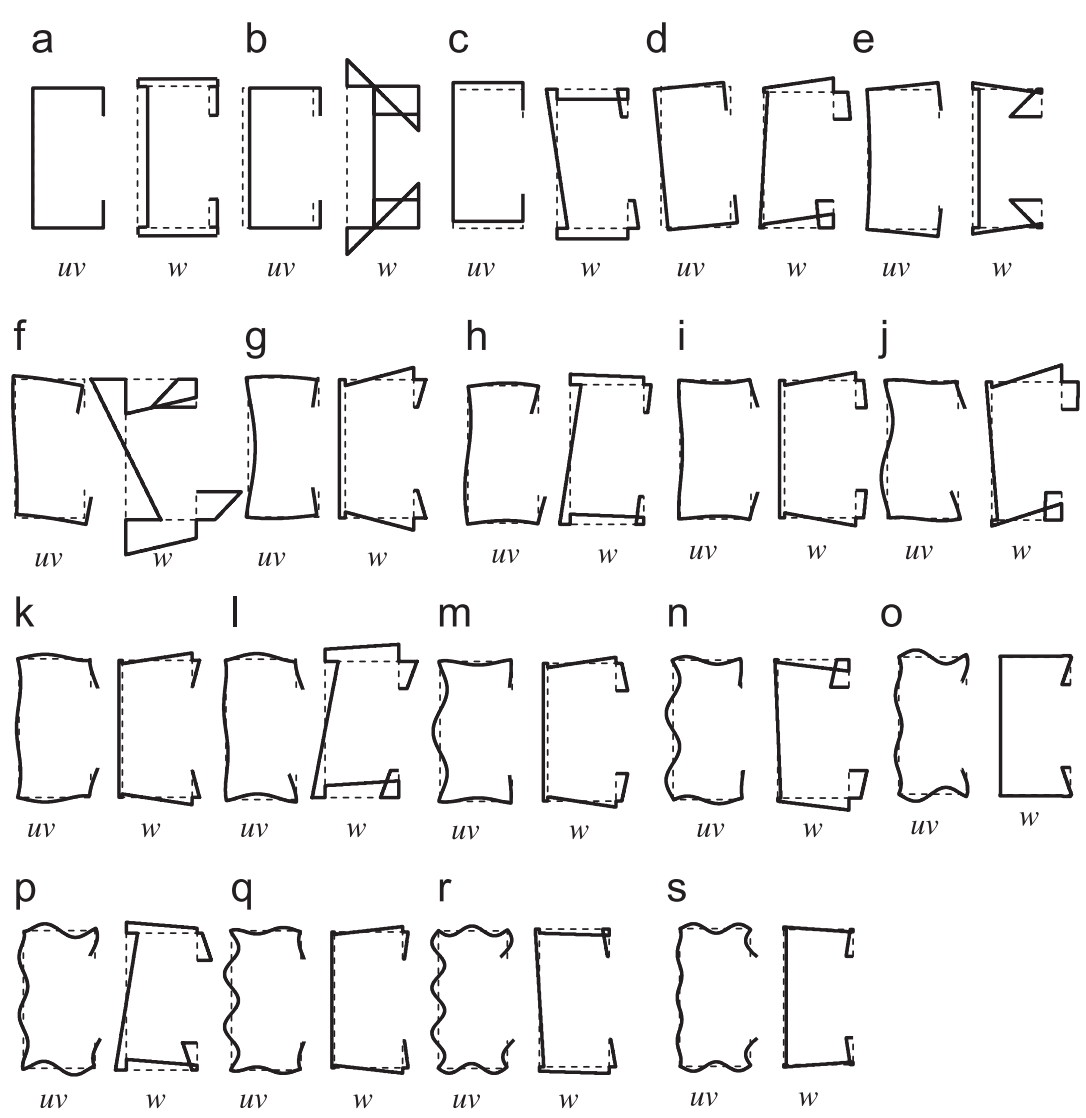

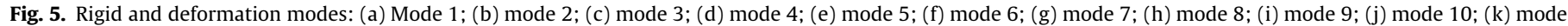
$11 ;(\mathrm{l})$ mode $12 ;(\mathrm{m})$ mode $13 ;(\mathrm{n})$ mode $14 ;(\mathrm{o})$ mode $15 ;(\mathrm{p})$ mode $16 ;(\mathrm{q})$ mode $17 ;(\mathrm{r})$ mode $18 ;(\mathrm{s})$ mode 19.

a

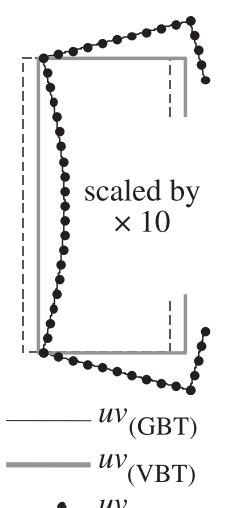

- $u v_{(\mathrm{ABAQUS})}$

d
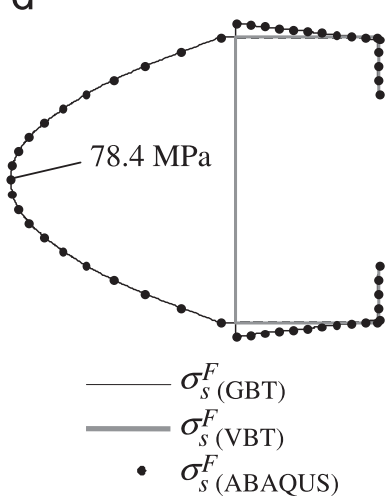

b

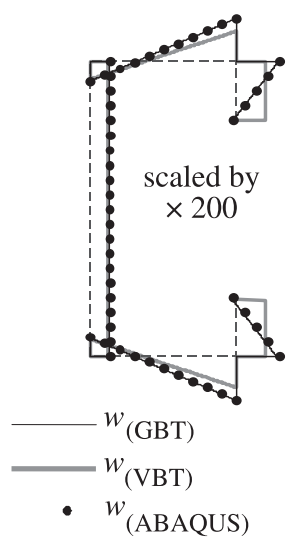

e

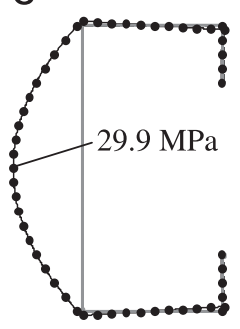

$\sigma_{z(\mathrm{GBT})}^{F}$ $\sigma_{z}^{F}$ (VBT)

- $\sigma_{z \text { (ABAQUS) }}^{F}$

C

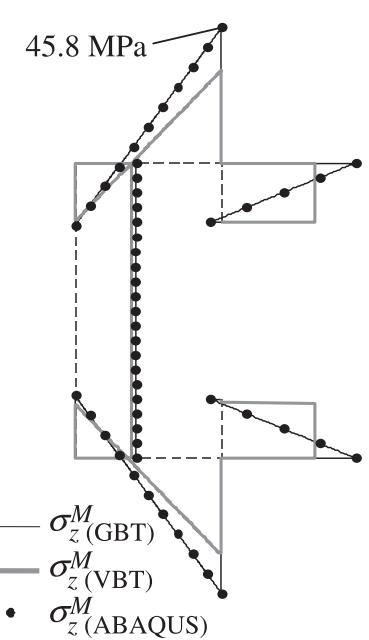

$f$

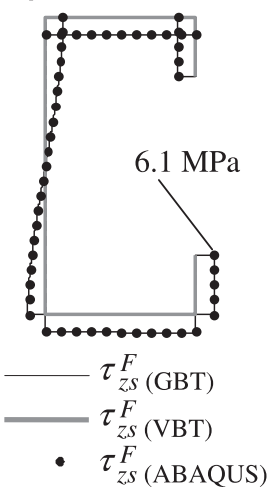

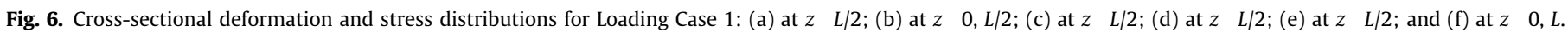



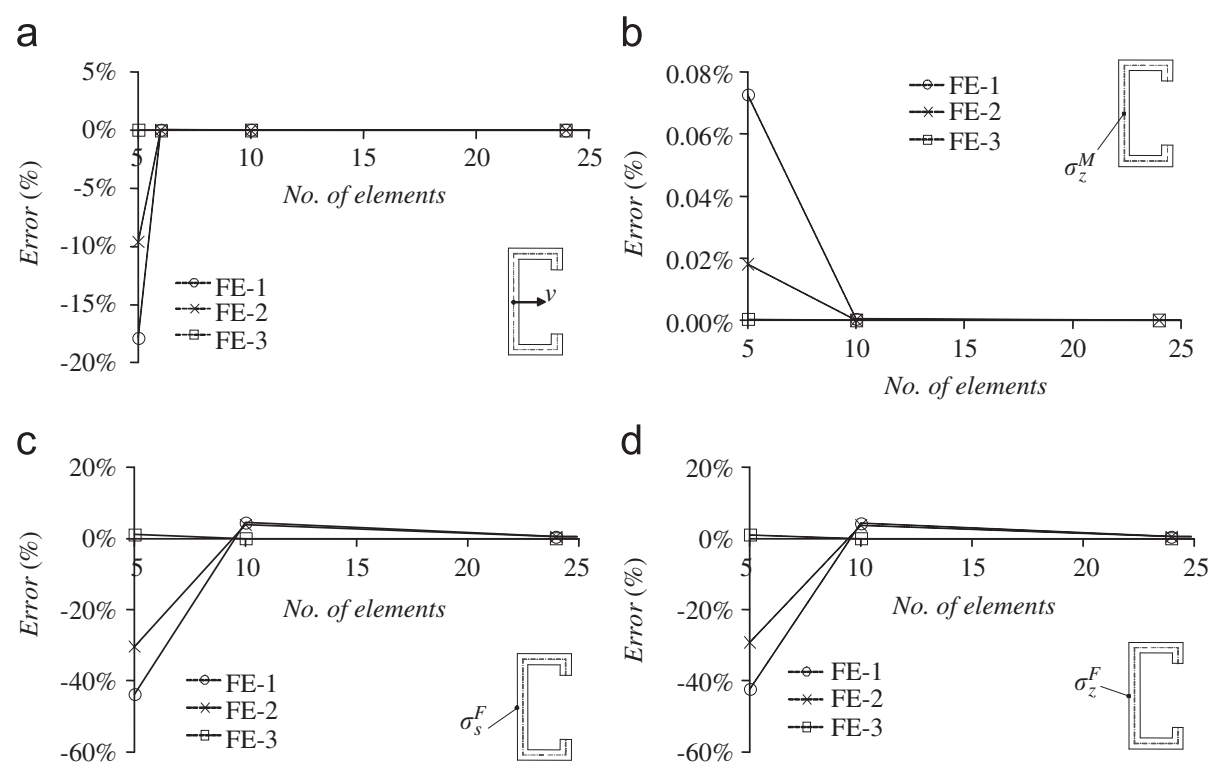

Fig. 7. Comparison between results obtained for Loading Case 1 using (i) FE-1: 6DOF with nodal masses, (ii) FE-2: 6DOF with consistent masses and (iii) FE-3: 8DOF with consistent masses: (a) deflections; (b) membrane stresses $\sigma_{z}^{M}$; (c) bending stresses $\sigma_{s}^{F}$; and (d) bending stresses $\sigma_{z}^{F}$.

a

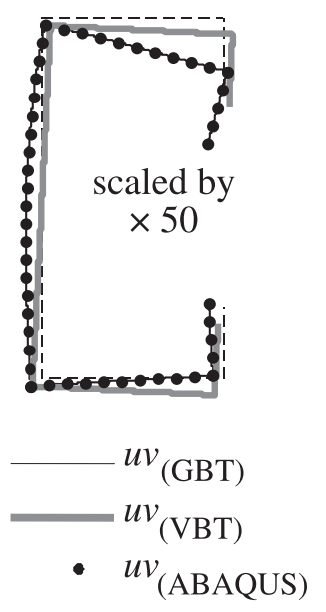

d

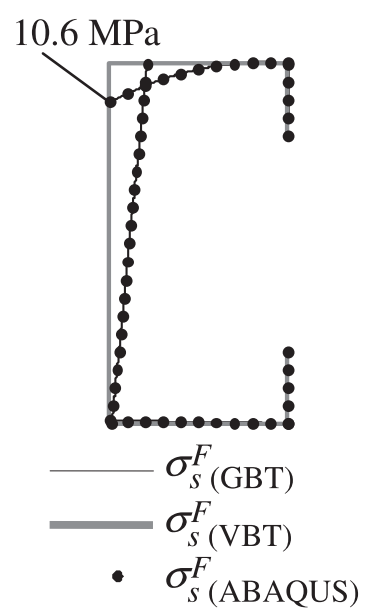

b
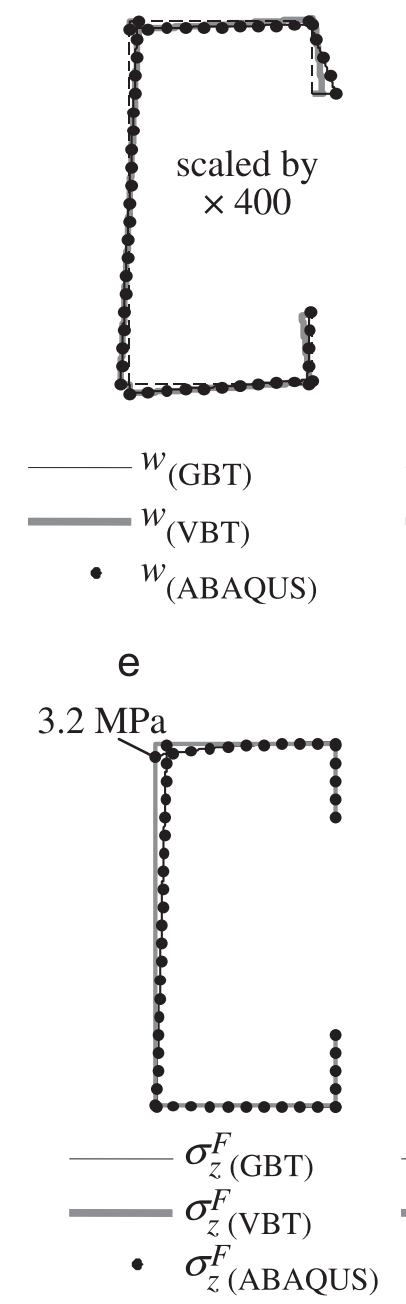

C

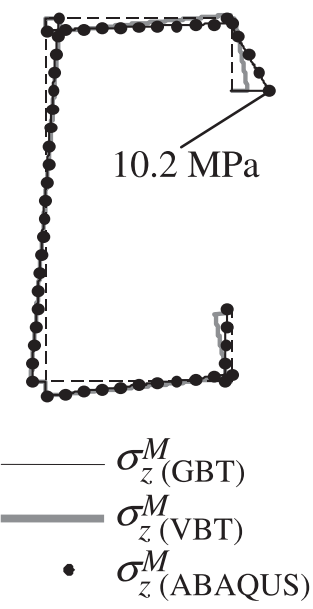

f

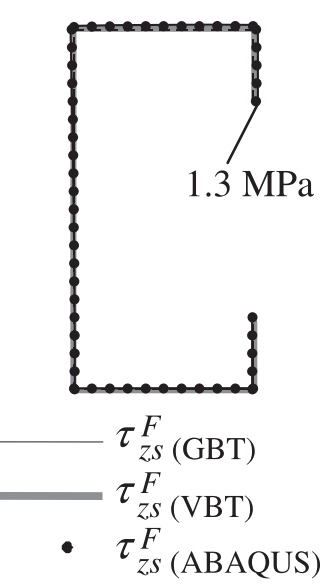

Fig. 8. Cross-sectional deformation and stress distributions for Loading Case $2:$ (a) at $z \quad L / 2$; (b) at $z \quad 0, L ;$ (c) at $z \quad L / 2$; (d) at $z \quad L / 2 ;$ (e) at $z \quad L / 2$; and (f) at $z \quad 0, L$. 
the 19 modes depicted in Fig. 5, have been presented in Fig. 6. The cross sectional and warping displacements have been scaled dif ferently to provide a clear overview of the deformed shapes. Also, all stresses have been plotted using the same scale to enable direct comparisons between their magnitudes. The GBT results have been compared against those furnished by the Vlasov Beam Theory (VBT), (which, in the spirit of the GBT, considers only the rigid modes of the cross section), and those obtained using a shell element model implemented in ABAQUS [41]. In general there is good agreement between the GBT and Abaqus results while, as expected, the VBT underestimates the deformation and stress states of the member. Modes 26 are the only ones with a participation greater than $1 \%$. This is calculated defining the participation of the $k$ th mode as the ratio between the maximum absolute value of its amplitude function and the sum of the maximum absolute values of the amplitude functions related to all modes considered in the analysis. In particular, the participations of the rigid modes 2, 3 and 4 are $5.3 \%, 9.1 \%$ and $59.9 \%$, respectively, and the ones of the deformable modes 5 and 6 equal 19.6\% and 4.5, respectively.

The performance of the three finite elements (i.e. previously referred to as FE 1, FE 2 and FE 3, respectively) to describe the deformation modes to be used for the cross sectional analysis has been compared in Fig. 7. This has been carried out considering only 9 deformation modes in the solution, which has been noted to produce errors within $1 \%$ for both deformations and stresses when compared to the results obtained using a large number of modes. In particular, different mesh refinements have been used to model the cross section. Based on these results, only FE 3 performs well with the coarser discretisation (i.e. with one FE element per plate segment) even if both FE 1 and FE 2 produce acceptable values (with an error of $4 \%$ when compared to the refined solution) for moderate levels of meshing. Fig. 7 highlights how flexural stresses are more sensitive to the number of finite elements specified and require higher levels of discretisation when using FE 1 and FE 2 to achieve an accurate solution.

The same lipped cross section has then been considered when subjected to a sinusoidal distributed load with amplitude $0.1 \mathrm{~N} /$ mm applied to its top flange (Fig. 4), referred to as Loading Case 2. Similarly to the previous case, the results calculated using the GBT have been obtained using the 19 modes illustrated in Fig. 5 and have been compared in Fig. 8 against those obtained with VBT and ABAQUS. Also in this case the GBT values match well with the ABAQUS ones. In this case, participations greater than $1 \%$ are exhibited by rigid mode 2 (with $37.8 \%$ ) and by the deformation modes 5 (with 53.7\%), 7 (with $7.2 \%$ ) and 9 (with $1.0 \%$ ).

When using the coarser mesh (i.e. one element per plate segment) the FE 3 produces errors of the order of $2 \%$ in the calculation of the stresses, when compared to the values obtained with highly refined meshes (Fig. 9). This error increases to about $8 \%$ and 20\% when using FE 1 and FE 2, respectively. For these a

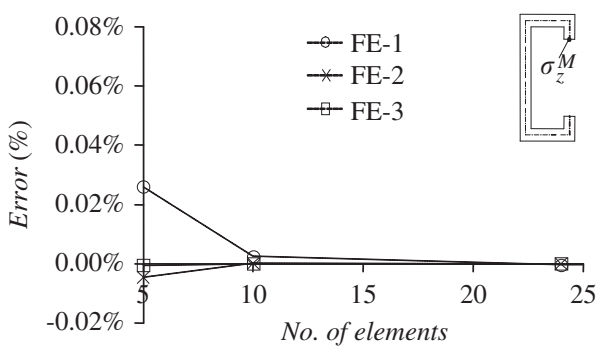

b

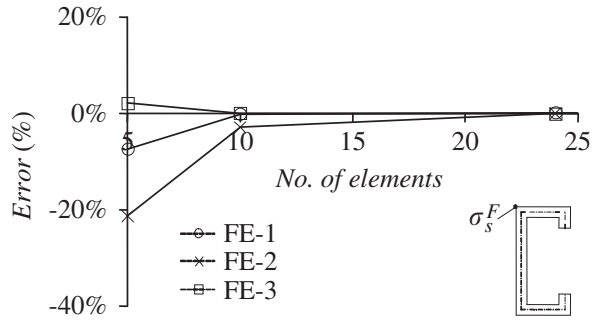

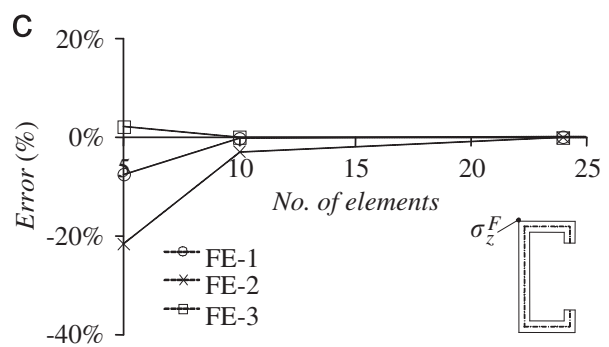

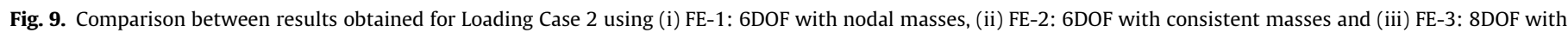
consistent masses: (a) membrane stresses $\sigma_{z}^{M}$; (b) bending stresses $\sigma_{s}^{F}$; and (c) bending stresses $\sigma_{z}^{F}$.

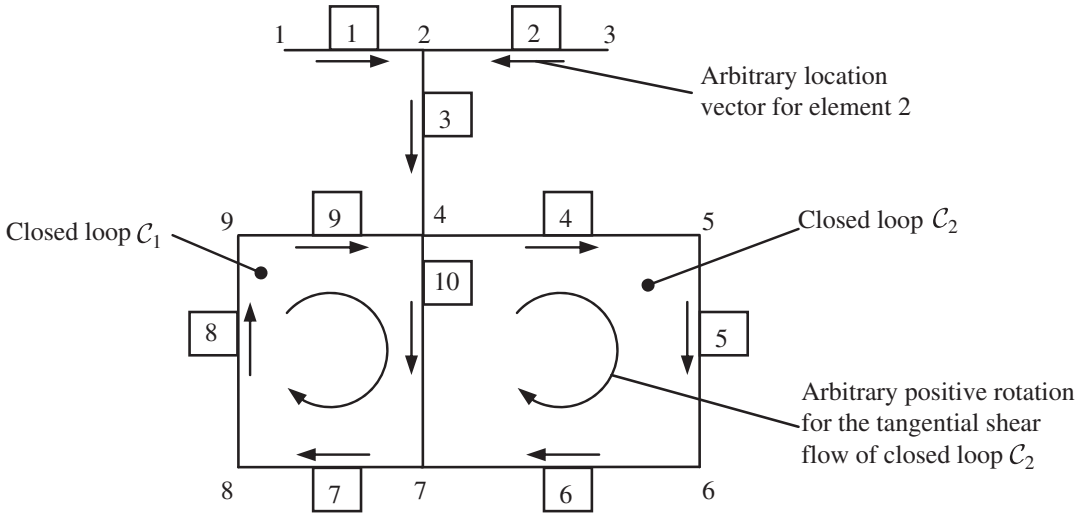

Fig. 10. Partially-closed cross-sections. 
latter two elements the error tends to decrease for moderate levels of meshing to a value of 3\% (Fig. 9).

\section{Conclusions}

A new approach has been presented for the determination of the conventional deformation modes within the framework of the Generalised Beam Theory (GBT), which is applicable to generic cross sections, i.e. open, partially closed and closed ones. The new method is based on a semi variational procedure, in which the in plane deformation modes are evaluated as the eigenfunctions of a positive semi definite auxiliary problem. This is chosen as the free dynamic problem of an unconstrained planar frame, repre senting the cross section of the structural member. Then, the resulting dynamic modes are adopted as the deformation modes for the GBT analysis, while the corresponding induced warping is calculated in a post processing phase, based on the enforcement of shear conditions. This procedure, reversed with respect the classical approach, heavily simplifies the cross sectional analysis of GBT and removes its need to separately determine bending, shear and local modes. Numerical applications have been pre sented to highlight the ease of use of the proposed approach. Moreover, an analytical worked example has been presented in Appendix A to better illustrate the determination of the warping displacements, consistently with the new cross sectional analysis.

\section{Acknowledgements}

The work of the first author was supported by the Australian Research Council through its Discovery Projects funding scheme (DP1096454).

\section{Appendix A}

The procedure proposed for the calculation of the warping functions can be applied once the functions $U_{k}$ and $V_{k}$ defining the deformations in the plane of the cross section are evaluated from the eigenvectors obtained from the dynamic analysis outlined in Section 3. The proposed process needs to be applied for each of the $K$ deformation modes considered (with $k=1, \ldots, K$ ). The unknown warping functions have been expressed in terms of their values at each node $W_{k}^{i}$ (with $i$ referring to the $i$ th node at which the warping function is calculated) in Eq. (22). Based on this, the unknowns to be determined consist of the warping nodal values $W_{k}^{i}$ and of the tangential shear flows $Q_{k}^{j}$ for each loop present in the section (with $j=1, \ldots J$ ). For illustrative purposes, the partially closed cross section depicted in Fig. 10 is considered, which is formed by 10 elements and 2 closed loops. In this case, the problem is setup in terms of 11 unknowns, which consist of the values of warping at each node $W_{k}^{i}$ (with $i=1, \ldots, 9$ ) and the values of the tangential shear flows $Q_{k}^{j}$ (with $j=1,2$ ) related to the $k$ th deformation mode considered (with $k=1, \ldots, K$ ).

The governing system of equations to be used for the 11 unknowns is obtained applying Eqs. (24) (26) as follows (with $i, j$, $e$ and $k$ being indexes used for nodes, closed loops, elements and deformation modes, respectively):

(i) Eq. (24) is applied to each plate element $e$ not included in a loop (i.e. free branched segment) in Fig. 10 there are 3 free branched segments and these correspond to elements $e=13$ :

$U_{k}^{e}{ }^{1}+\frac{W_{k}^{i}{ }^{2} W_{k}^{i} \quad 1}{b^{e}{ }^{1}}=0$

$U_{k}^{e} \quad{ }^{2}+\frac{W_{k}^{i}{ }^{2} W_{k}^{i} \quad 3}{b^{e}{ }^{2}}=0$
$U_{k}^{e} \quad{ }^{3}+\frac{W_{k}^{i}{ }^{4} W_{k}^{i} \quad 2}{b^{e}{ }^{3}}=0$

(ii) Eq. (26) is written for each plate element $e$ part of a loop there are 7 plate segments in Fig. 10, which satisfy this condition and consist of elements $e=410$ :

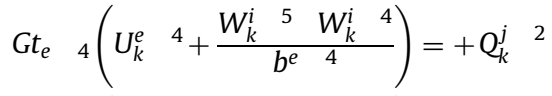

$$
\begin{aligned}
& G t_{e} \quad 5\left(\begin{array}{llll}
U_{k}^{e} & 5 \\
& +\frac{W_{k}^{i}}{}{ }^{6} & W_{k}^{i} & 5 \\
b^{e} & 5
\end{array}\right)=+Q_{k}^{j} \quad 2
\end{aligned}
$$

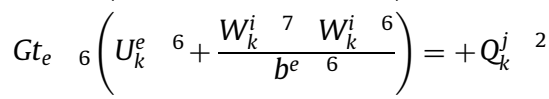

$$
\begin{aligned}
& G t_{e} \quad 7\left(\begin{array}{llll}
U_{k}^{e} & 7 \\
& +W_{k}^{i}{ }^{8} & W_{k}^{i} & 7 \\
b^{e} & 7
\end{array}\right)=+Q_{k}^{j} \quad 1
\end{aligned}
$$

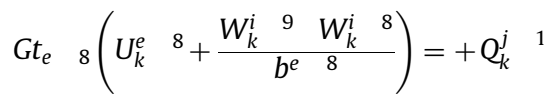

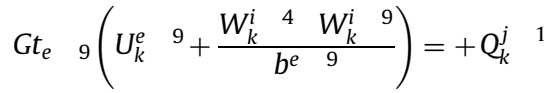

$$
\begin{aligned}
& G t_{e} \quad 10\left(\begin{array}{llll}
U_{k}^{e} & 10
\end{array}\right.
\end{aligned}
$$

(iii) Eq. (25) is applied considering every plate element $e$ forming the cross section for the section of Fig. 10 the contribu tion of all 10 elements is considered:

$$
\begin{aligned}
& \frac{W_{k}^{i}{ }^{2}+W_{k}^{i}{ }^{1}}{2} b^{e}{ }^{1}+\frac{W_{k}^{i}{ }^{2}+W_{k}^{i}{ }^{3}}{2} b^{e}{ }^{2}+\frac{W_{k}^{i}{ }^{4}+W_{k}^{i}{ }^{2}}{2} b^{e} \quad{ }^{3} \\
& +\frac{W_{k}^{i}{ }^{5}+W_{k}^{i} \quad 4}{2} b^{e} \quad 4 \\
& +\frac{W_{k}^{i}{ }^{6}+W_{k}^{i}{ }^{5}}{2} b^{e}{ }^{5}+\frac{W_{k}^{i}{ }^{7}+W_{k}^{i} \quad{ }^{6}}{2} b^{e} \quad{ }^{6} \\
& +\frac{W_{k}^{i}{ }^{8}+W_{k}^{i}{ }^{7}}{2} b^{e} \quad 7 \\
& +\frac{W_{k}^{i}{ }^{9}+W_{k}^{i}{ }^{8}}{2} b^{e}{ }^{8}+\frac{W_{k}^{i}{ }^{4}+W_{k}^{i}}{2} b^{e} \quad 9 \\
& +\frac{W_{k}^{i}{ }^{7}+W_{k}^{i}{ }^{4}}{2} b^{e} \quad{ }^{10}=0
\end{aligned}
$$

\section{References}

[1] Schardt R. Verallgemeinerte Technicsche Biegetheory. Germany: SpringlerVerlag; 1989.

[2] Schardt R. Generalised beam theory-an adequate method for coupled stability problems. Thin-Walled Structures 1994;19:161-80.

[3] Davies JM, Leach P. First-order generalised beam theory. Journal of Constructional Steel Research 1994;31:187-220.

[4] Davies JM, Leach P, Heinz D. Second-order generalised beam theory. Journal of Constructional Steel Research 1994;31:221-41.

[5] Leach P. The calculation of modal cross-section properties for use in the generalised beam theory. Thin-Walled Structures 1994;19:61-79.

[6] Leach P, Davies JM. An experimental verification of the generalised beam theory applied to interactive buckling problems. Thin-Walled Structures 1996;25(1):61-79.

[7] Jiang C, Davies JM. Design of thin-walled purlins for distortional buckling. Thin-Walled Structures 1997;29(1-4):189-202.

[8] Concalves R, Ritto-Correa M, Camotim D. A new approach to the calculation of cross-section deformation modes in the framework of generalised beam theory. Computational Mechanics 2010;46(5):759-81.

[9] Dinis PB, Camotim D, Silvestre N. FEM-based analysis of the local-plate/ distortional mode interaction in cold-formed steel lipped channel columns. Computers and Structures 2007;85:1461-74.

[10] Silvestre N, Camotim D. GBT buckling analysis of pultruded FRP lipped channel members. Computers and Structures 2003;81:1889-904.

[11] Silvestre N, Camotim D. First-order generalised beam theory for arbitrary orthotropic materials. Thin-Walled Structures 2002;40:755-89.

[12] Dinis PB, Camotim D, Silvestre N. GBT formulation to analyse the buckling behaviour of thin-walled members with arbitrary 'branched' open crosssections. Thin-Walled Structures 2006;44:20-38. 
[13] Goncalves R, Dinis PB, Camotim D. GBT formulation to analyse the first-order and buckling behaviour of thin-walled members with arbitrary cross-sections. Thin-Walled Structures 2009;47:583-600.

[14] Silvestre N. Generalised beam theory to analyse the buckling behaviour of circular cylindrical shells and tubes. Thin-Walled Structures 2007;45:185-98.

[15] Camotim D, Silvestre N, Basaglia C, Rebiano R. GBT-based buckling analysis of thin-walled members with non-standard support conditions. Thin-Walled Structures 2008;46:800-15.

[16] Silvestre N, Camotim D. Asymptotic-numerical method to analyse the postbuckling behaviour, imperfection-sensitivity, and mode interaction in frames. Journal of Engineering Mechanics ASCE 2005;131(6):617-32.

[17] Basaglia C, Camotim D, Silvestre N. GBT-based buckling analysis of thinwalled steel frames with arbitrary loading and support conditions. International Journal of Structural Stability and Dynamics 2010;10(3):363-85.

[18] Basaglia D, Camotim D, Silvestre N. Global buckling analysis of plane and space thin-walled frames in the context of GBT. Thin-Walled Structures 2008;46:79-101.

[19] Silvestre N. Buckling behaviour of elliptical cylindrical shells and tubes under compression. International Journal of Solids and Structures 2008;45:4427-47.

[20] Goncalves R, Camotim D. GBT local and global buckling analysis of aluminium and stainless steel columns. Computers and Structures 2004;82:1473-84.

[21] Silvestre N, Camotim D. Distortional buckling formulae for cold-formed steel $\mathrm{C}$ and $\mathrm{Z}$ section members part I-derivation. Thin-Walled Structures 2004;42:1567-97.

[22] Silvestre N, Camotim D. Second-order generalised beam theory for arbitrary orthotropic materials. Thin-Walled Structures 2002;40:791-820.

[23] Rebiano R, Silvestre N, Camotim D. Local and global vibration of thin-walled members subjected to compression and non-uniform bending. Journal of Sound and Vibration 2008;315:509-35.

[24] Silvestre N, Camotim D. Vibration behaviour of axially compressed coldformed steel members. Steel and Composite Structures 2006;6(3):221-36.

[25] Silvestre N, Camotim D. Nonlinear generalised beam theory for cold-formed steel members. International Journal of Structural Stability and Dynamics 2003;3(4):461-90.
[26] Silvestre N, Camotim D. Local-plate and distortional postbuckling behaviour of cold-formed steel lipped channel columns with intermediate stiffeners. Journal of Structural Engineering ASCE 2006;132(4):529-40.

[27] Silvestre N, Young B, Camotim D. Non-linear behaviour and load-carrying capacity of CFRP-strengthened lipped channel steel columns. Engineering Structures 2008;30:2613-30.

[28] Adany S, Schafer BW. Buckling mode decomposition of single-branched open cross-section members via finite strip method: derivation. Thin-Walled Structures 2006;44:563-84.

[29] Adany S, Schafer BW. Buckling mode decomposition of single-branched open cross-section members via finite strip method: application and examples. Thin-Walled Structures 2006;44:585-600.

[30] Casafront M, Marimon F, Pastor MM. Calculation of pure distortional elastic buckling loads of members subjected to compression via the finite element method. Thin-Walled Structures 2009;47(6-7):701-29.

[31] Vlasov VZ. Thin-Walled Elastic Beams, Monson; 1961.

[32] Cheung YK. Finite Strip Method in Structural Analysis.Pergamon Press; 1976.

[33] Cheung YK, Fan SC, Wu CQ. ) Spline finite strip in structural analysis. In: Proceedings of the International Conference on Finite Element Method, Shanghai, China; 1982.

[34] Cheung YK, Tham LG, Li WY. Free vibration and static analysis of general plate by spline finite strip. Computational Mechanics 1988;3:187-97.

[35] Cheung YK, Lam LG. The Finite Strip Method.CRC Press; 1997.

[36] Friedrich R. Finite strip method: 30 years-a bibliography (1968-1998). Engineering Computations 2000;17(1):92-111.

[37] Bathe KJ. Finite Element Procedures.Prentice Hall; 2006.

[38] Cook RD, Malkus DS, Plesha ME, Witt RJ. Concepts and Applications of Finite Element Analysis. 4th editionjohn Wiley \& Sons; 2002.

[39] Chopra AK. Dynamics of Structures: Theory and Applications to Earthquake Engineering. 2nd editionPrentice Hall; 2001.

[40] Adany S, Schafer BW. A full model decomposition of thin-walled, singlebranched open cross-section members via the constrained finite strip method. Journal of Constructional Steel Research 2008;64(1):12-29.

[41] ABAQUS. Standard User's Manual Version 6.8-1, Hibbitt, Karlsson and Sorensen, Inc, Vol 1-3, USA; 2008. 